\title{
Alternative right ventricular assist device implantation technique for patients with perioperative right ventricular failure
}

\author{
Diyar Saeed, MD, PhD, Bujar Maxhera, MD, Hiroyuki Kamiya, MD, PhD,
} Artur Lichtenberg, MD, PhD, and Alexander Albert, MD, PhD

\section{ABSTRACT}

Objectives: Temporary right ventricular assist devices (RVADs) may be required to support patients with perioperative refractory right ventricular failure (RVF). We report on our experience using a different technique of RVAD implantation that does not necessitate resternotomy at the time of RVAD removal.

Methods: Patients with perioperative RVF who underwent temporary RVAD implantation between January 2010 and February 2014 were reviewed. A dacron graft was attached to the pulmonary artery and passed through a subxiphoid exit, where the RVAD outflow cannula was inserted. The inflow cannula was percutaneously cannulated in the femoral vein, and the sternum was primarily closed. On the day of RVAD explantation, the outflow graft of the RVAD was pulled and ligated, and the insertion site was secondarily closed. The RVAD inflow cannula was removed, and direct pressure was applied.

Results: Twenty-one patients (age $58 \pm 14$ years) were supported. Seventeen patients $(81 \%)$ had RVF after left ventricular assist device implantation, and 4 patients developed postcardiotomy RVF. The median duration of RVAD support was 9 days (range: $2-88$ days). Eleven patients (52\%) were successfully weaned from the RVAD. Two patients were bridged to transplantation. Eight patients died on left ventricular assist device and/or RVAD support. The survival rates to discharge or heart transplantation, and to 1 -year, were $62 \%$ and $52 \%$, respectively.

Conclusions: No technical issues were encountered in this large series of RVAD implantations using the described technique for various forms of postoperative RVF. Extended support duration and reduction of resternotomy risks may be the main advantages of this technique compared with conventional RVAD implantation methods. (J Thorac Cardiovasc Surg 2015;149:927-32)

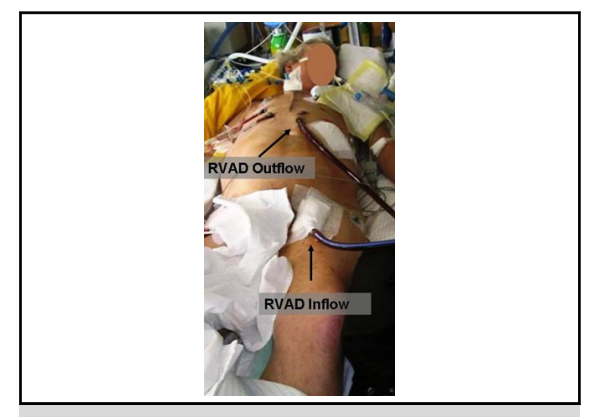

Example of a patient with simultaneous LVAD and RVAD support

\section{Central Message}

This study reports the feasibility of an alternative technique of RVAD implantation in patients with perioperative right ventricular failure that does not necessitate resternotomy at the time of RVAD removal. Extended support duration and reduction of resternotomy risks are the main advantages of this technique compared to conventional techniques.

\section{Author Perspective}

In patients with perioperative right ventricular failure (RVF), many surgeons implant a temporary RVAD or ECMO by using open sternotomy approach. The main drawback of these techniques is the higher incidence of bleeding and infection or the necessity for resternotomy if the sternum is closed. In this study, we are reporting our experience and the feasibility of a less invasive technique of RVAD implantation for RVF that does not necessitate resternotomy at the time of RVAD removal. Twentyone patients were supported. Seventeen patients had RVF after LVAD implantation and 4 patients developed postcardiotomy RVF. No technical issues were encountered.

See Editorial Commentary pages 933-4
Perioperative right ventricular failure (RVF) is a devastating clinical condition with a high mortality rate that sometimes necessitates mechanical support. ${ }^{1,2}$ A clinically significant RVF occurs in about $0.1 \%$ of patients after cardiac surgery. The incidence is higher, reaching 2\%-3\%, after

\footnotetext{
From the Clinic for Cardiovascular Surgery, Heinrich-Heine University of Düsseldorf, Düsseldorf, Germany.

Read (in part) at the 27th Annual Meeting of The European Association for CardioThoracic Surgery, Vienna, Austria, October 5-9, 2013.

Received for publication July 30, 2014; revisions received Sept 11, 2014; accepted for publication Oct 26, 2014; available ahead of print Nov 26, 2014

Address for reprints: Diyar Saeed, MD, PhD, Department of Cardiovascular Surgery, University Hospital of Düsseldorf, Heinrich-Heine University, Moorenstraße 5, 40225 Düsseldorf, Germany (E-mail: diyar.saeed@med.uni-duesseldorf.de). $0022-5223 / \$ 36.00$

Copyright (c) 2015 by The American Association for Thoracic Surgery http://dx.doi.org/10.1016/j.jtcvs.2014.10.104
}

heart transplantation, and can reach 20\%-30\% after left ventricular assist device (LVAD) implantation. ${ }^{3,4}$

Several right ventricular assist device (RVAD) implantation techniques have been described. ${ }^{5-8}$ In the context of postcardiotomy RVF, many surgeons tend to use extracorporeal membrane oxygenation (ECMO) and leave the sternum open for a few days until the right ventricle has fully recovered. In patients with RVF after LVAD implantation, many surgeons implant a temporary RVAD through an open sternotomy approach using direct right atrial and pulmonary artery cannulation.

The main drawback of these techniques is the higher incidence of bleeding and infection when the chest remains unclosed, and the necessity for resternotomy if the sternum is closed at the time of the primary operation. Several new approaches, designed to avoid these complications, have been 


\section{Abbreviations and Acronyms \\ $\mathrm{CPB}=$ cardiopulmonary bypass \\ $\mathrm{CT}=$ computed tomography \\ $\mathrm{ECMO}=$ extracorporeal membrane oxygenation \\ LVAD $=$ left ventricular assist device \\ RVAD $=$ right ventricular assist device \\ $\mathrm{RVF}=$ right ventricular failure}

described for temporary RVAD implantation. The majority of descriptions are anecdotal case reports, and the approaches have been used mainly after LVAD implantation..$^{8-12}$ In this study, we aimed to report our experience using a less-invasive technique of RVAD implantation for RVF after various cardiac surgical procedures that does not necessitate resternotomy at the time of RVAD removal.

\section{MATERIALS AND METHODS \\ Patient Population}

The study protocol was approved by the local research ethics board. A retrospective data review was performed from January 2010 through February 2014. Inclusion criteria included having undergone RVAD implantation performed, using the technique described in the next section, after various forms of postcardiotomy RVF. Patients with veno-arterial ECMO support for postcardiotomy RVF, subjects intended for long-term biventricular support, and those who underwent direct open cannulation of the pulmonary artery were excluded from this study. The preoperative characteristics of the patients, and postoperative outcomes, are described in Table 1.

\section{Right Ventricular Assist Device Implantation \\ Technique}

The criteria for RVAD support after LVAD implantation at our institution include an echocardiographic finding of severe right ventricular dysfunction combined with central venous pressure $>20 \mathrm{~mm} \mathrm{Hg}$, mean arterial pressure of $<60 \mathrm{~mm} \mathrm{Hg}$, and a low pump index of $<2.21 / \mathrm{min} / \mathrm{m}^{2}$ on a more-thanmoderate dose of inotropic support (epinephrine and milrinone).

The implantation was performed in all patients using a median sternotomy approach, which was necessary for the primary operation. A QuickDraw (22-25 French) (Edwards Lifesciences, Irvine, Calif) or Bio-Medicus Multi-Stage (23-25 French) venous cannula (Medtronic, Inc, Minneapolis, Minn) was used as inflow and was inserted percutaneously into the right atrium through the right femoral vein using the Seldinger technique. An $8-\mathrm{mm}$ or $10-\mathrm{mm}$ dacron graft (Gelweave; Vascutek, Ltd, Inchinnan, Renfrewshire, Scotland) was anastomosed end-to-side to the main pulmonary artery with a $5 / 0$ polypropylene running suture, using a Satinsky side clamp. The outflow graft was passed transcutanously through a separate opening on the right subcostal margin, where the outflow cannula was inserted-a Fem-Flex II (21-23 French) (Edwards Lifesciences, Irvine, Calif) or an HK 36 PB-Maquet (GmbH \& Co KG, Rastatt, Germany).

The graft was tied tightly around the cannula outside the chest and secured firmly to the chest wall with multiple sutures; the chest was primarily closed in all cases. Figure 1 shows a patient with simultaneous LVAD and RVAD support. In 1 patient, RVAD implantation for RVF was necessary 1 day after LVAD implantation, which was implanted through a J-sternotomy approach. Therefore, the RVAD implantation was performed using the same J-sternotomy approach (Figure 2).

The RVAD perfusion system consisted of a centrifugal pump. A Levitronix CentriMag (Levitronix GmbH, Zurich, Switzerland) was used as an RVAD in all cases. For patients with additional hypoxia, a pressure- controlled biocompatible heparin-coated silicon-membrane oxygenator, the Hilite 7000 LT (Medos Medizintechnik AG, Stolberg, Germany) or the EO 5019 (EUROSETS s.r.1., Medolla, Italy), and a heat exchanger were used. Based on our experience with noncontinuous biventricular assist device support, ${ }^{13}$ the RVAD flow was adjusted to be $10 \%-20 \%$ lower than the LVAD flow in patients with LVAD support. A left atrial catheter was inserted into the left atrium in all cases to continuously monitor left atrial pressure and prevent overdriving of the RVAD flow.

Anticoagulation with heparin was started 24 hours after the surgery, with a target-activated partial thromboplastin time of 50-60 seconds. Additionally, a daily dose of $100 \mathrm{mg}$ of acetylsalicylic acid was given from the first postoperative day. Anticoagulation was withheld in patients with active bleeding. In patients with LVAD, coumadin with a target international normalized ratio of 2-3 was started after removal of the chest tubes.

\section{Right Ventricular Assist Device Explantation Technique}

Removal of the RVAD support was performed after gradual reduction of the RVAD flow to $2 \mathrm{~L} / \mathrm{min}$ at 0.5 -L/day increments under echocardiography guidance. Meanwhile, mild doses of inotropic and phosphodiesterase inhibitor agents were initiated. RVAD removal was considered if central venous pressure was $<15 \mathrm{~mm} \mathrm{Hg}$, mean arterial pressure was $>60 \mathrm{~mm} \mathrm{Hg}$, and cardiac index and/or pump index remained $2.4 \mathrm{~L} / \mathrm{min} / \mathrm{m}^{2}$. The skin exit site of the graft was widely prepared and draped. Gentle traction on the graft allowed the redundant portions of the graft inside the chest to be exposed. The graft was clamped and oversewn, and multiple heavy ligators were applied. Finally, the graft was reinserted into the chest, and the skin incision was closed. Removal of the inflow femoral cannula was performed after application of a deep U-stich with manual compression of the groin insertion site.

\section{Statistical Analysis}

The statistical analysis was performed using SPSS 16.0 (SPSS, Chicago, Ill). Values of continuous data are presented as mean \pm standard deviation, or as median with interquartile range when appropriate. Categoric variables are displayed as frequency distributions (n) and simple percentages $(\%)$. Survival rate was calculated with the Kaplan-Meier product-limit estimator.

\section{RESULTS}

A total of 63 LVADs were implanted during this time period. Temporary RVAD using the described technique was performed in 17 patients $(27 \%)$. The right ventricular function of these patients was considered severely depressed before LVAD implantation. Meanwhile, 4 patients underwent RVAD implantation for postcardiotomy RVF and were included in this study. Table 1 shows the patients' characteristics.

The postcardiotomy RVF patients included 2 patients with RVF after aortocoronary bypass surgery; 1 patient with acute RVF after aortic root replacement; and 1 patient with acute RVF after aortic root replacement and aortocoronary bypass surgery. The right ventricular function of these patients, except for 1, was documented as normal before the primary surgery. The reason for the RVF in patients with aortic root surgery may have been kinking of the right coronary artery. Fifteen patients $(71 \%)$ underwent RVAD implantation at the same time as primary surgery. The mean time between primary surgery and RVAD implantation in the remaining 6 patients was $2.7 \pm 2.7$ days (range, $2-8$ days). 
TABLE 1. Perioperative characteristics of the patients

\begin{tabular}{lc}
\hline \multicolumn{1}{c}{ Characteristics and outcomes } & Mean \pm SD or n (\%) \\
$\mathbf{N}=\mathbf{2 1}$ \\
\hline Age (y) & $58 \pm 14$ \\
Male gender & $15(71)$ \\
Postcardiotomy cardiogenic shock & $4(19)$ \\
After LVAD implantation & $17(81)$ \\
Primary diagnosis & \\
ICM & $12(57)$ \\
DCM & $5(24)$ \\
AMI & $4(19)$ \\
Pre-RVAD IABP & $6(29)$ \\
Pre-RVAD ECMO & $13(62)$ \\
Redo surgery & $4(19)$ \\
Oxygenator requirement & $12(57)$ \\
Mediastinal bleeding requiring resternotomy & $6(29)$ \\
Sternal wound infection & $3(14)$ \\
Outcome & \\
Weaned & $11(52)$ \\
Died & $8(38)$ \\
Received transplant & $2(10)$ \\
\hline
\end{tabular}

$S D$, Standard deviation; $L V A D$, left ventricular assist device; $I C M$, ischemic cardiomyopathy; $D C M$, dilative cardiomyopathy; $A M I$, acute myocardial infacrtion; $R V A D$, right ventricular assist device; $I A B P$, intra-aortic balloon pump; $E C M O$, extracorporeal life support.

The RVAD implantation was performed using cardiopulmonary bypass $(\mathrm{CPB})$ machine in 14 patients, with LVAD support for 3 patients, ECMO support for 3 patients, and off-pump in 1 patient. Twelve patients $(57 \%)$ required an oxygenator in addition to the RVAD at the time of LVAD/RVAD implantation. All these patients had ECMO support before LVAD/RVAD implantation. The median duration of RVAD support was 9 days (range, 2-88 days). The longest duration of support was in a 51-year-old patient with ischemic cardiomyopathy who underwent simultaneous LVAD/RVAD implantation, with transplantation performed after 88 days of support. ${ }^{14}$ Eleven patients $(52 \%)$, were successfully weaned from the RVAD, which was removed. Two patients were bridged directly to heart transplantation after 8 and 88 days of support, respectively. Eight patients (38\%) died on LVAD and/or RVAD support.

Postoperative complications include respiratory failure in 15 patients $(71 \%)$, and renal failure in 11 patients $(52 \%)$ (respiratory and renal failure were defined per the Interagency Registry for Mechanically Assisted Circulatory Support definition). Bleeding that necessitated resternotomy occurred in 6 patients $(29 \%)$. Superficial sternal wound infection was observed in 2 patients and was treated conservatively. In 1 patient, severe mediastinitis was diagnosed several days after LVAD and/or RVAD implantation and after RVAD removal. This patient was treated with intermittent vacuum-assisted closure therapy, ultimately underwent a heart transplantation, and died several days after the heart transplantation. In this patient, the sternum was left open for 7 days after aorto-coronary bypass surgery and

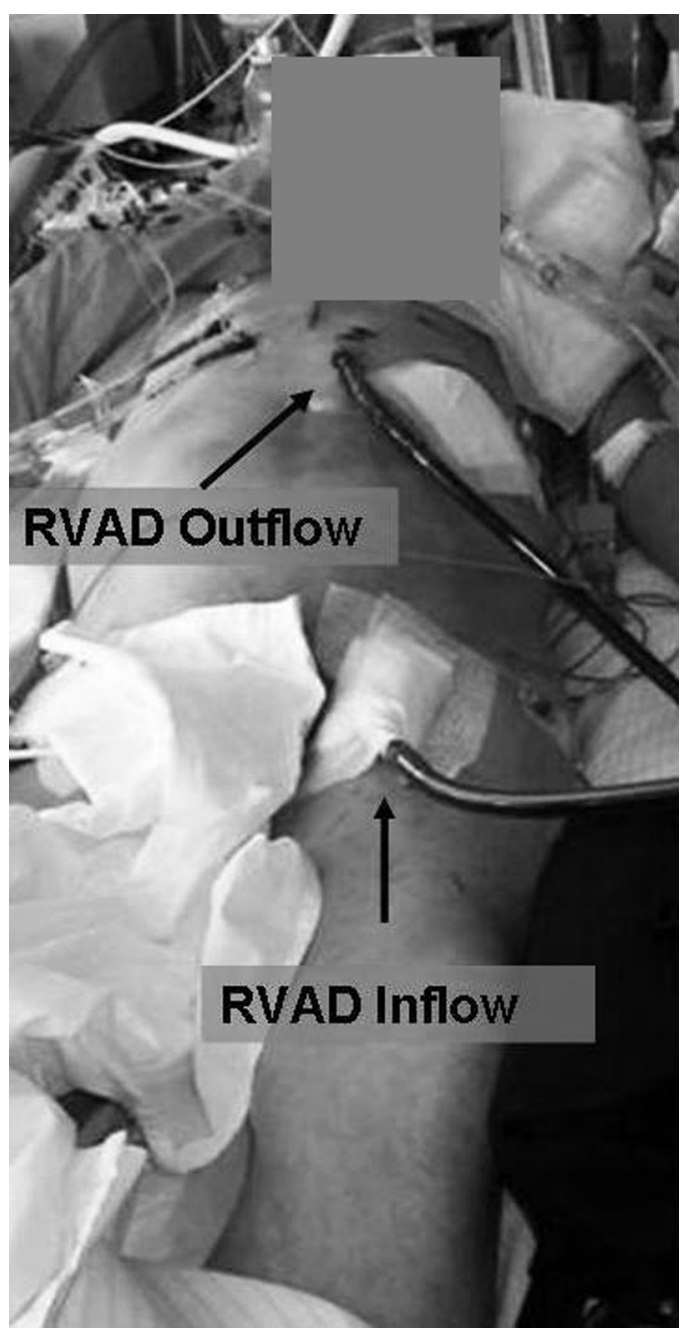

FIGURE 1. A patient with simultaneous LVAD and RVAD support. The inflow cannula of the RVAD was inserted percutaneously into the right atrium through the left femoral vein. The outflow cannula was inserted into a graft that was attached to the pulmonary artery and exteriorized through the subxyphoid area. RVAD, Right ventricular assist device.

ECMO implantation before LVAD and RVAD implantation. No stroke or pulmonary embolism event was documented in any of these patients.

The survival rate to discharge or heart transplantation was $62 \%$, and the 1 -year survival rate was $52 \%$. The 1 year survival rate in the postcardiotomy patients was better $(75 \%)$ than that for patients requiring RVAD support after LVAD implantation $(47 \%)(P=.25)$ (Figure 3$)$. Causes of death included septicemia in 4 patients, refractory hepatic failure in 2 patients, hemorrhagic shock in 1 patient, and severe intestinal ischemia in 1 patient. Another 3 patients died at 42, 91, and 226 days after RVAD explantation, respectively. The causes of late mortality in these patients were cardiogenic shock after heart transplantation, congestive heart failure, and severe, chronic, obstructive airway disease, respectively. 


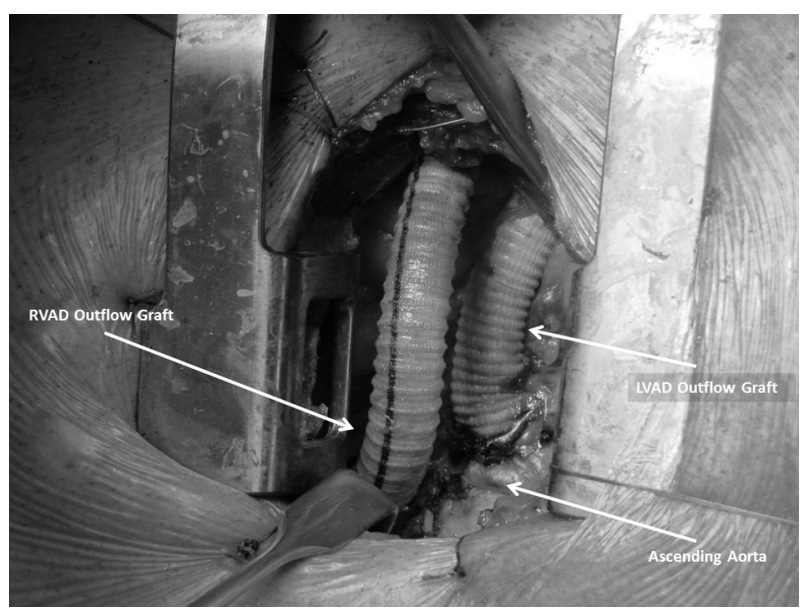

FIGURE 2. A patient with simultaneous LVAD and RVAD support implanted throughuse of a J-sternotomy approach. The inflow cannula of the RVAD was inserted percutaneously into the right atrium through the right femoral vein. The outflow cannula was inserted into a graft that was attached to the pulmonary artery and exteriorized through the subxyphoid area. $R V A D$, Right ventricular assist device; $L V A D$, left ventricular assist device.

\section{DISCUSSION}

This article shows the feasibility and applicability of the described RVAD implantation technique, which does not necessitate resternotomy at the time of RVAD removal, for various durations of support, after various forms of perioperative RVF. No technical issues were encountered in any of these patients in the course of RVAD support.

When conservative measures for RVF fail to adequately improve right ventricular function and provide adequate organ perfusion, mechanical support of the right ventricle is inevitable. One available option is the implantation of a peripheral veno-arterial ECMO. ${ }^{5,10}$ However, ECMO,

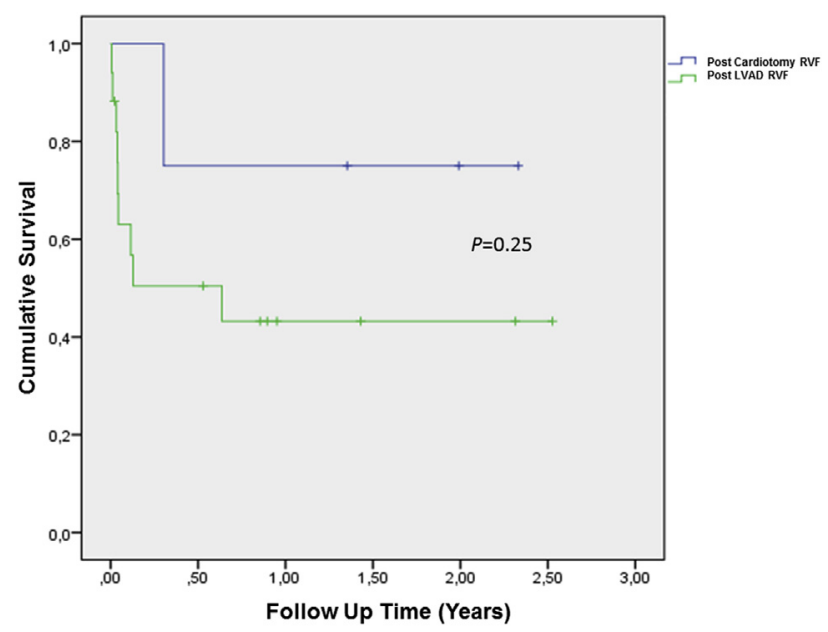

FIGURE 3. Kaplan-Meier survival curve of the patients. The survival rate to discharge/heart transplantation in this study was $62 \%$. The one year survival in the postcardiotomy patients was better $(75 \%)$ than patients requiring RVAD support following LVAD implantation $(47 \%)(P=.25)$. $R V F$, Right ventricular failure; $L V A D$, left ventricular assist device. although suitable for cardiopulmonary support in some instances, has a higher rate of device-related complications (thromboembolism, hemolysis, ischemic leg complications, and bleeding) with longer duration of support, which may be necessary in some instances, as it was for 1of our patients who needed 88 days of support. Furthermore, in a recently published study from Noly and colleagues,${ }^{15} 10$ patients were treated for RVF after LVAD implantation using a femoro-femoral veno-arterial ECMO, and were compared with another 8 patients who underwent RVAD implantation using the technique described in their article. A better outcome and fewer thromboembolic events were observed when RVAD was implanted using femoral vein and pulmonary artery cannulation, as described in the current article.

Another RVAD support technique has been reported by Loforte and colleagues. ${ }^{7}$ The authors attached a graft to the pulmonary artery and inserted a 2-stage venous cannula as an inflow cannula into the right atrium, and extended it through the chest. The main advantages of this technique include easier mobilization of the patient, because groin cannulation is avoided. The main drawback of this technique is the necessity of reoperation at the time of RVAD removal.

The feasibility of another novel technique of percutaneous RVAD implantation was postulated by Columbia University colleagues. ${ }^{8}$ However, the limited number of patients and experience with this technique precludes a wide application of this technology for a longer duration at this stage of development. Cheung and colleagues ${ }^{16}$ recently published their experience with the Impella right direct and right peripheral temporary ventricular assist devices (Abiomed, Inc, Danvers, Mass). A total of 18 patients were supported for up to 19 days with a 1-year survival rate of $50 \%$. The main advantage of Impella use is the possibility of peripheral application without sternotomy in cases in which the right peripheral Impella can be used (only 3 patients in the Cheung and colleagues ${ }^{16}$ study). However, the limited experience with this device, and the shorter support duration, do not allow wide application of this promising technology at the current time.

The rationale for adopting this technique for temporary RVAD implantation is to reduce morbidity and possibly the mortality rate after RVAD separation. Our proposed technique foremost allows for a simplified RVAD explantation, without the need for resternotomy, that can even be performed at the bedside in the intensive-care unit.

Postoperative bleeding is a known complication in patients with RVF who require RVAD support. A high reexploration rate for bleeding of $39 \%$ has been previously reported. ${ }^{15}$ Among the other advantages of this technique may be the reduced rate of bleeding, owing to early sternal closure. In this study, 6 patients $(29 \%)$ required re-exploration for bleeding, 5 of whom were LVAD patients. The relatively high rate of RVAD implantation in our long-term LVAD population $(30 \%)$ may be explained 
by the fact that $76 \%$ of LVAD populations in this series were critically sick patients who were on ECMO support at the time of LVAD implantation and the lower threshold for RVAD implantation at our center.

An additional advantage of this technique includes the possibility of integrating an oxygenator with the RVAD circuit in case of hypoxia or severe lung edema. This advantage is particularly helpful in patients with lung injury who are on ECMO and require long-term LVAD support. ${ }^{17}$ In our study, 12 patients $(57 \%)$ required an oxygenator in addition to the RVAD at the time of LVAD and/or RVAD implantation. All of these patients had ECMO support before LVAD and/or RVAD implantation. Notably, cases with significant pulmonary valve insufficiency need to be excluded from consideration for use of this RVAD technique. All of these patients underwent pre- and intra-operative transesophageal echocardiography, and cases with more-thanmoderate pulmonary valve insufficiency were excluded.

This technique of RVAD implantation may be performed using CPB, on ECMO support, on LVAD support, or even off-pump if the patient can tolerate the partial clamping of the pulmonary artery. In this study, RVAD implantation was performed using CPB in 14 patients: on LVAD support only in 3 patients, on ECMO support in 3 patients, and off-pump in 1 patient. The current strategy in our center involves LVAD implantation on ECMO, without using $\mathrm{CPB}$, in the absence of aortic valve insufficiency and/or patent foramen ovale. The feasibility of long-term RVAD support using this technique is another advantage of this RVAD implantation technique. The median duration of RVAD support in this study was 9 days (range, 2-88 days).

This technique allows early extubation. However, the main drawback of the technique is limited mobilization, owing to the presence of the inflow cannula in the groin. The majority of the patients remained bed- or chairbound. Three patients in this study were extubated after several days of support, and full mobilization was performed in only 1 patient, with prolonged support duration of 88 days. This patient was able to walk with RVAD in place, and had already been upgraded to a high-urgency state. We therefore decided to forego an additional permanent extracorporeal RVAD implantation procedure and bridge to heart transplantation.

The potential for microbial colonization of the vascular graft, and subsequent deep-seated mediastinitis, is a possibility, but this complication can be avoided through careful use of aseptic techniques. In addition, retracting the conduit subcutaneously reduces the infection risk. In this series, 3 patients $(14 \%)$ developed superficial wound infection with no signs of graft infection; they were treated conservatively, except for 1 patient who developed severe mediatinitis. Furthermore, no concerns have been raised so far regarding the possibility of graft infection in other small series in which this technique was used. ${ }^{9,11,18}$

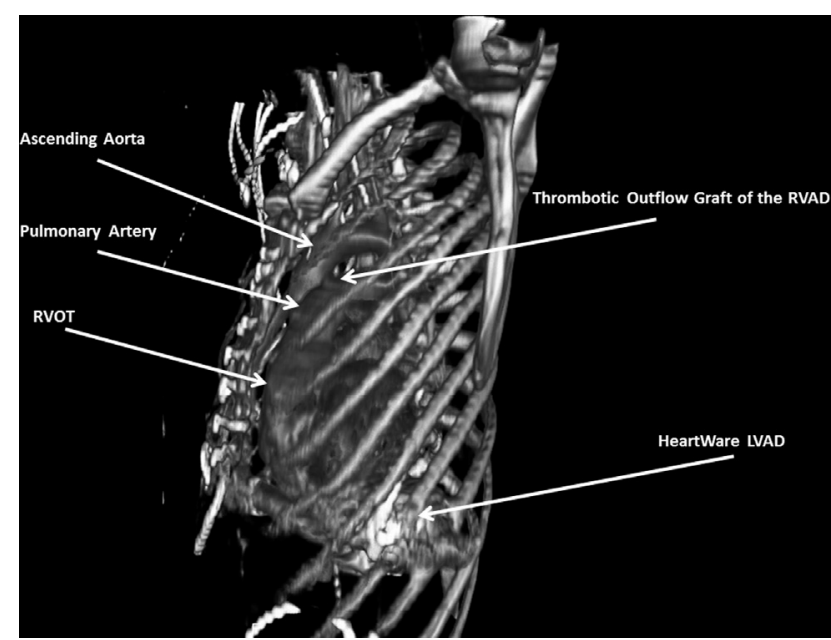

FIGURE 4. A 3-dimensional computed tomography scan of another LVAD patient after RVAD explantation. HeartWare LVAD (HeartWare, Framingham, Mass) is shown. RVAD, Right ventricular assist device; RVOT, right ventricular outflow tract; $L V A D$, left ventricular assist device.

There may be some theoretical risks of intermittent pulmonary embolism from thrombotic graft after RVAD removal. We have not performed regular computed tomography (CT) scans in these patients to look specifically for pulmonary embolism. However, none of the patients developed clinical signs of pulmonary embolism during support. Some of our patients did have CT scans, for various reasons, and these confirmed the thrombotic closure of the graft and excluded pulmonary embolism. A 3-dimensional reconstruction of the CT scan was performed in one of the patients (Figure 4). An occluded outflow graft can be observed. In another study, from the Cleveland Clinic, ${ }^{18}$ using a similar form of RVAD implantation technique in 10 patients, CT scans were performed in all patients after hospital discharge, to assess the retained outflow graft. In that study, thrombotic grafts were demonstrated at a mean of 148 days without pseudoaneurysm or peri-graft fluid collections, which further confirms the safety and efficacy of this technique.

This technique is not new and has been described before in patients with pulsatile LVADs, and in anecdotal case reports including only a few patients. ${ }^{12,18,19}$ However, the current study is of particular interest because it reports on a large series of patients who were supported with RVAD, using this technique as a "proof of concept." Moreover, the study does not include only LVAD patients, as $19 \%$ of the patients in this cohort underwent RVAD implantation for postcardiotomy RVF, which otherwise would have been treated conventionally using a veno-arterial ECMO system.

Various survival rates after treatment with the Levitronix CentriMag RVAD (Levitronix GmbH, Zurich, Switzerland) have been reported, ranging from $0 \%$ to $58 \% .^{20-22}$ All of these studies used different forms of implantation technique that necessitated reoperation for RVAD removal. The survival rate to discharge or heart transplantation in this 
study was $62 \%$, and 1 -year survival was $52 \%$, which is quite satisfactory compared with the previously mentioned studies. Figure 3 shows the Kaplan-Meier survival curve of the patients. A trend toward better 1-year survival in the postcardiotomy patients $(75 \%)$ was observed, compared with patients requiring RVAD support after LVAD implantation $(47 \%)(P=.25)$. However, the limited number of supported patients in the postcardiotomy arm precludes further conclusions from this study. Survival rate remains limited after RVAD implantation in LVAD patients, regardless of the implantation technique used. ${ }^{16}$ This finding further confirms the negative influence of RVF on overall outcome, even in the era of nonpulsatile pumps.

We emphasize the importance of using a continuous left atrial-pressure monitoring line in these patients, adjusting the LVAD flow so it is $10 \%$ to $20 \%$ higher than the RVAD flow, and performing regular chest radiographs to avoid iatrogenic lung edema, which is a possibility when this technique is used. The limitations of this study include its retrospective nature and the small number of patients. This study does not compare the technique to other alternatives for RVF, such as ECMO, conventional cannulation, or permanent RVAD. Furthermore, no data are available regarding the echocardiographic right ventricular measurements at various times after RVAD implantation. In addition, Swan-Ganz catheters (Hemex Scientific Inc, Austin, Tex) were not used exclusively during and after RVAD support for all patients. Therefore, these data are missing in this study. The main limitation of this technique was the restricted mobility of the patient owing to groin cannulation. Further potential complications of this technique include pulmonary embolism and later graft infection.

\section{CONCLUSIONS}

This study shows the feasibility and safety of the proposed RVAD implantation technique for various forms of perioperative RVF. A satisfactory outcome can be achieved with minimal rate of complications. Early extubation, extended support duration, and reduction of resternotomy risks may be the main advantages of this technique. The RVAD removal can be reproducibly performed under minimal anesthesia and without the need for resternotomy. The survival rate remains limited in patients requiring RVAD support after LVAD implantation.

\section{Conflict of Interest Statement}

Authors have nothing to disclose with regard to commercial support.

\section{References}

1. Buckberg G, Hoffman JI. Right ventricular architecture responsible for mechanical performance: unifying role of ventricular septum. J Thorac Cardiovasc Surg. 2014;148:3166-71.

2. Aissaoui N, Morshuis M, Schoenbrodt M, Hakim Meibodi K, Kizner L, Börgermann J, et al. Temporary right ventricular mechanical circulatory support for the management of right ventricular failure in critically ill patients. $J$ Thorac Cardiovasc Surg. 2013;146:186-91.

3. Haddad F, Couture P, Tousignant C, Denault AY. The right ventricle in cardiac surgery, a perioperative perspective: II. Pathophysiology, clinical importance, and management. Anesth Analg. 2009;108:422-33.

4. Kaul TK, Fields BL. Postoperative acute refractory right ventricular failure: incidence, pathogenesis, management and prognosis. Cardiovasc Surg. 2000;8:1-9.

5. Dalén M, Sartipy U, Lund LH, Fux T, Corbascio M, Svenarud P, et al. Peripheral extracorporeal membrane oxygenation as short-term right ventricular support after heartware left ventricular assist device implantation. ASAIO J. 2013;59: 523-5.

6. Griffith KE, Jenkins E, Stulak J, Paugh T, Pagani FD. Long-term use of the Cen$\operatorname{triMag}(\mathrm{R})$ Ventricular Assist System as a right ventricular assist device: a case report. Perfusion. 2012;27:65-70.

7. Loforte A, Montalto A, Lilla Della Monica P, Musumeci F. Simultaneous temporary CentriMag right ventricular assist device placement in HeartMate II left ventricular assist system recipients at high risk of right ventricular failure. Interact Cardiovasc Thorac Surg. 2010;10:847-50.

8. Takayama H, Naka Y, Kodali SK, Vincent JA, Addonizio LJ, Jorde UP, et al. A novel approach to percutaneous right-ventricular mechanical support. Eur J Cardiothorac Surg. 2012;41:423-6.

9. Strauch JT, Franke UF, Madershahian N, Wahlers T. Right ventricular assist device implantation - a new transcutaneous approach. Thorac Cardiovasc Surg. 2004;52:378-9.

10. Kai M, Tang GH, Malekan R, Lansman SL, Spielvogel D. Venoarterial extracorporeal membrane oxygenation for right heart failure complicating left ventricular assist device use. J Thorac Cardiovasc Surg. 2014;147:e31-3.

11. Haneya A, Philipp A, Puehler T, Rupprecht L, Kobuch R, Hilker M, et al. Temporary percutaneous right ventricular support using a centrifugal pump in patients with postoperative acute refractory right ventricular failure after left ventricular assist device implantation. Eur J Cardiothorac Surg. 2012;41: 219-23.

12. De Silva RJ, Soto C, Spratt P. Extra corporeal membrane oxygenation as right heart support following left ventricular assist device placement: a new cannulation technique. Heart Lung Circ. 2012;21:218-20.

13. Saeed D, Ootaki Y, Ootaki C, Akiyama M, Horai T, Catanese J, et al. Acute in vivo evaluation of an implantable continuous flow biventricular assist system. ASAIO J. 2008;54:20-4.

14. Ortmann P, Saeed D, Lichtenberg A. Case report of extended "temporary" use of Levitronix CentriMag right ventricular assist device. Artif Organs. 2012;36: 1072-3.

15. Noly PE, Kirsch M, Quessard A, Leger P, Pavie A, Amour J, et al. Temporary right ventricular support following left ventricle assist device implantation: a comparison of two techniques. Interact Cardiovasc Thorac Surg. 2014;19:49-55.

16. Cheung AW, White CW, Davis MK, Freed DH. Short-term mechanical circulatory support for recovery from acute right ventricular failure: clinical outcomes. J Heart Lung Transplant. 2014;33:794-9.

17. Boulate D, Luyt CE, Pozzi M, Niculescu M, Combes A, Leprince P, et al. Acute lung injury after mechanical circulatory support implantation in patients on extracorporeal life support: an unrecognized problem. Eur J Cardiothorac Surg. 2013;34:544-9.

18. Shafii AE, Chamogeorgakis T, Mountis M, Gonzalez-Stawinski G. Fate of retained right ventricular assist device outflow grafts after right ventricular recovery. J Heart Lung Transplant. 2012;31:672-3.

19. Haj-Yahia SM, Birks EJ, Hardy J, Yacoub MH, Khaghani A. Minimally invasive technique for explantation of right ventricular assist devices. Ann Thorac Surg. 2006;82:1547-8.

20. Bhama JK, Kormos RL, Toyoda Y, Teuteberg JJ, McCurry KR, Siegenthaler MP. Clinical experience using the Levitronix CentriMag system for temporary right ventricular mechanical circulatory support. J Heart Lung Transplant. 2009;28:971-6.

21. John R, Long JW, Massey HT, Griffith BP, Sun BC, Tector AJ, et al. Outcomes of a multicenter trial of the Levitronix CentriMag ventricular assist system for shortterm circulatory support. J Thorac Cardiovasc Surg. 2011;141:932-9.

22. Shuhaiber JH, Jenkins D, Berman M, Parameshwar J, Dhital K, Tsui S, et al. The Papworth experience with the Levitronix CentriMag ventricular assist device. J Heart Lung Transplant. 2008;27:158-64.

Key words: right ventricle, circulatory support devices (LVAD, RVAD, BVAD, TAH), cardiomyopathy, adult, heart failure 